Title: Kayaking performance is altered in mentally fatigued young elite athletes Paper code: J Sports Med Phys Fitness-9051

Submission Date: 2018-06-07 23:29:21

Article Type: Original Article

Files:

1) : Manuscript

Version: 2

Description: Manoscritto originale

File format: application/msword

2) : Figures 1

Version: 1

Description: Figura 1

File format: image/tiff

3): Figures 2

Version: 1

Description: Figura 2

File format: image/tiff

4): Figures 3

Version: 1

Description: Figura 3

File format: image/tiff

5): Figures 4

Version: 1

Description: Figura 4

File format: image/tiff

6): Figures 5

Version: 1

Description: Figurd 5

File format: image

7): Figures 6

Version: 1

Description: Mryera 6

File foxmat

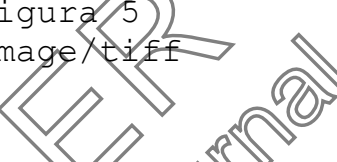

(O)

(3)

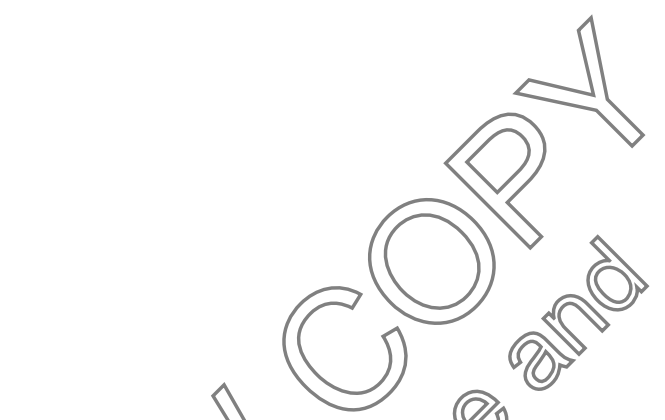




\section{Kayaking performance is altered in mentally fatigued young elite athletes}

Walter Staiano, Andrea Bosio*, Gaia Piazza, Marco Romagnoli, Pietro L. Invernizzi

Walter Staiano, Faculty of Science of Physical Education and Sport, University of Valencia, Spain

Andrea Bosio (corresponding author), Human Performance Laboratory, Mapei Spprt, Italy

Via Busto Fagnano, 38, 21057 Olgiate Olona (VA), Italy.

Telephone +390331575757 Fax +390331575700 e-mail: andrea.bosid@mapeisport.it

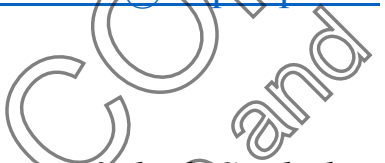

Gaia Piazza, Department of Biomedical Sciences for Health, Universitä deg(1) Studi di Milano, Italy

Marco Romagnoli, Faculty of Science of Physical Educationnat Sport, University of Valencia, Spain

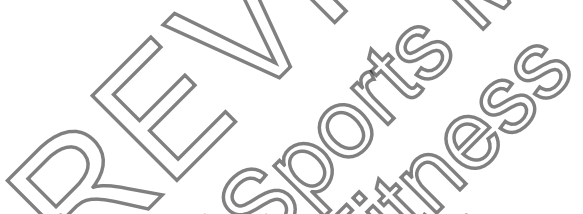

Pietro L. Invernizzi, Department of Biomedical Seences for Health, Università degli Studi di Milano, Italy

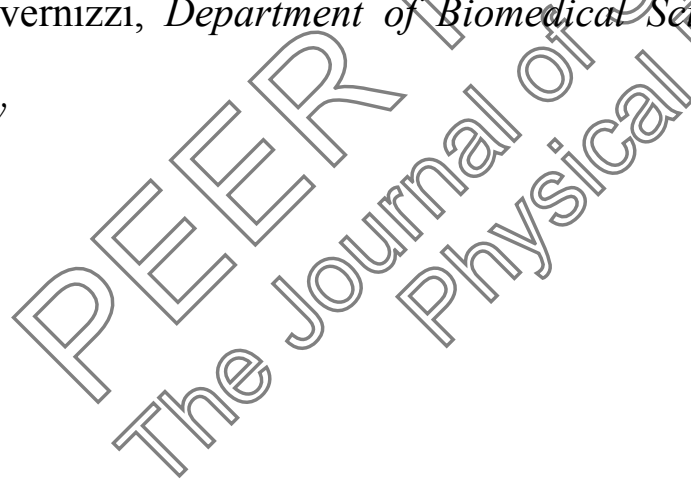




\begin{abstract}
The present study aimed to assess the impact of 60 min of a cognitive demanding task inducing mental fatigue (Stroop) on kayaking performance in young elite athletes. The second objective was to elucidate the effect of mental fatigue on performance in a population of young under-17 elite athletes of national. Thirteen under-17 elite kayakers completed $60 \mathrm{~min}$ of an incongruent Stroop color-word test, or the equivalent time in a control condition in a cross-over study design. Afterwards, participants completed a $2000 \mathrm{~m}$ kayaking time trial in which power output, stroke rate and time at the end of 400, 800, 1200, 1600 and $2000 \mathrm{~m}$ were recorded Physiologeat and perceptual measures of heart rate (HR), blood lactate and rating of perceised exertion (RPE) were collected during the time trial. Psychological questionnaires were used to assess fatigue and mental demand of the Stroop. Subjective ratings of mental fatigue following the Stroop were almost certainly higher. Results of the time suggested that mental fatigue almost

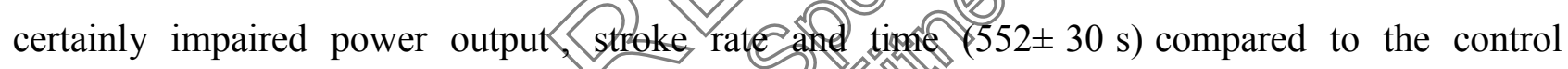
$(521 \pm 36 \mathrm{~s})$ condition. Yet, during the time tral RPE was almost certainly higher in the mental fatigue condifion, while HRowas reported to be possibly lower. Blood lactate resulted almost certainly lowent the mental fatigue state at completion of the time trial $\left(10.3 \pm 1.5 \mathrm{vs} 12.2 \pm 1.6 \mathrm{mmgl} \cdot \mathrm{L}^{1}\right)$. In conclusion, mental fatigue has an almost certain negative impact on 2000-m Kayaking performance in young elite athletes.
\end{abstract}

Key words: cognition, perception, RPE, endurance performance, mental fatigue. 


\section{Introduction}

In the past decade in the sport world, it has drastically increased the attention and the interest for cognitive processes relating to sport performance ${ }^{1}$. The brain has begun to play such a central role as ultimate challenge in the sport domain that it has pushed exercise scientists to expand and combine their knowledge with other areas of expertise such as neurophysiology ${ }^{1}$.

Particular attention have been given to the role that mental fatigue has on subsequent physical performance ${ }^{2}$. Defined as a psychobiological state caused by prolonged periods of demanding cognitive activity ${ }^{3}$, mental fatigue has been proved to produce a deleterious effect on several types of physical exercises ${ }^{2}$. Endurance performance ${ }^{3-5}$ as well as internittent running and repeated sprint ability ${ }^{6}$ studies have revealed the negative impact that pre iously induced mental fatigue has on physical exercise. Instead, mental fatigue does notsseems to altenamerobic and maximal power/force performance ${ }^{7}$.

Despite the significant detrimental impact that mental farigue can have on the physical domain, it is still debated and often overlooked its importance in thespert comfext and more in particular in the elite world. This may be due to the fact that previons researein involved mainly sedentary or welltrained participants ${ }^{2,8}$. Very few studies hấne used elite or experienced athletes as participants. Martin et $\mathrm{al}^{7}$ was the frist to investigate, elife athletes would be affected differently by mental fatigue compared to anateurs in aycling trime trial. In that study it has been revealed that elite cyclists were more resilient and resist more to mental fatigue compared to amateur ones when the cognitive stimulus induing mental fatigue was $30 \mathrm{~min}^{\circ}$ long ${ }^{7}$. It was hypothesized that elite athletes are characterized by a higher inhibitory control that might be both genetically in origin and/or developed during many years of sport practice.

It is not clear whether young elite athletes behave similarly and possess the same attitude. However, very recently it has been questioned whether a given cognitive task (of a certain duration) is sufficient to induce significant mental fatigue ${ }^{8}$. Therefore, very little is known on the effect of 
mental fatigue on particular population such as elite athletes. Furthermore research conducted so far has mainly focused on cycling, running, swimming and football performance, while other sports (both team and individual), characterized by an endurance component, still lack of evidence upon the detrimental effect that mental fatigue can have on specific performance.

The amount of controlled studies testing the effects of mental fatigue on performance is still scarce and even reviews and meta-analysis carried out thus far cannot clearly show the net detrimental effect of mental fatigue ${ }^{8}$. Therefore, more research and even replication studies seem to be necessary to increase the knowledge in this field.

Kayak is a sport characterised by exceptional demands on upper body performance and the importance of the aerobic and anaerobic system is very well knøwn 2, 10 In fact. Several studies suggest that Olympic kayak paddlers not only need a high aerobic power, but the anaerobic contribution is also very important for successful performanee 11,12 Many studies have been conducted identifying the physical demand of this sport ${ }^{9}$ However no studies have been previously conducted on the effect of mentâfatigue on this particular sport where a combination of

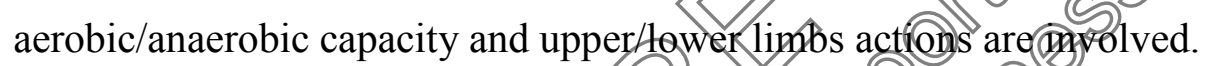

The main aim of this study was to test the hypothesis that 60 min of a highly demanding cognitive task inducing mental fatigue produces a reduction simulated $2000 \mathrm{~m}$ kayaking performance test in young elite athletes.

Moreover, the second aim of this tuay was to determine if mental fatigue would affect performance in a population of young imder-17 elite athletes of national level. As pointed out in Martin et al.'s study ${ }^{7}$, elite athletes were immune to 30 min of a mentally demanding cognitive task probably due to the amount of physical and cognitive training they were exposed during many years. In the present study we investigated if the assumption proposed by Martin at al. ${ }^{7}$ are still valid even in young under 17 athletes.

\section{Methods}




\section{Participants}

Thirteen elite kayakers from the under -17 national team $(16.4 \pm 0.8$, years, $60.7 \pm 7.0 \mathrm{~kg}, 172.5 \pm 7.2$ $\mathrm{cm}, \sim 5$ training sessions per week, an average of 5 years of kayaking experience) signed an informed consent form describing the study protocol and possible discomfort which was approved by the Institutional Review Board of the department where the study was conducted according to the Declaration of Helsinki for the Human Rights. Given the age of participants a parent or the responsible of the parental authority for each participant also signed the informed consent. All participants received written instructions describing study procedures but werenaive to its true aims and hypotheses. At the end of the final visit, participants were debriefeenand asked not to discuss the real aims of the study with other participants.

\section{Experimental Protocol}

A randomised crossover design was used for the present stad.5.) The order of the experimental treatment (mental fatigue/control or control mental fatiguedwas allocated in random order based on

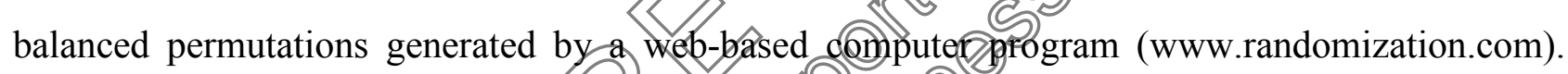
Participants were required to visit the laboratery on 3 codsions, in a period no longer than 2 weeks between the first and lastyisit. Testing during visige, 2 and 3 (experimental visits) was completed at the same time of the day. The first visit ated as familiarization in which participants became familiar with the Stroop tash and athe psychological, perceptual and physiological measures. During visits 2 and 3 araticipants completed the baseline mood and motivation questionnaire, followed by either the Stroop task or the control task. Afterwards they filled in the subjective workload and again the mood questionnaires. Participants were then moved to the kayak ergometer where they completed a standardized warm-up and a $2000 \mathrm{~m}$ time trial.

Prior to visits 2 and 3, participants were instructed to drink $35 \mathrm{ml}$ of water per kilogram of body weight, sleep for at least $7 \mathrm{~h}$, refrain from the consumption of alcohol, and avoid any vigorous exercise the day before visiting the laboratory. Participants were also instructed to avoid any 
caffeine and mentally demanding tasks for at least $3 \mathrm{~h}$ before testing. The day of visit 2, participants were asked to record the time and content of the meals consumed before testing, and to keep them consistent the day of visit 3 . At the beginning of visits 2 and 3, participants were asked to complete a checklist to ascertain that they had complied with the instructions given to them. Participants were also asked to declare if they had taken any medication/drug or had an acute illness, injury, or infection on the day.

\section{Experimental treatment}

In the mental fatigue condition participants completed a 60 -min modifiedincongruent version of the Stroop colour-word task. Participants performed this cognitive task at a eomprat, whilst sitting comfortably in a quiet, dimly lit room. This Stroop task consists of four words (yellow, blue, green, red) serially presented on the computer screen, displayed until the participant responded, followed by a $1.5 \mathrm{~s}$ rest interval. Participants were instructed to press pnesof four coloured buttons on the keyboard (yellow, blue, green, red), with the correct respoinge being the button corresponding to the ink colour (either yellow, blue, green,red) of the wodp preseneed on the screen. For example, if the word blue appeared in yellow ink, the yellow button had to be pressed. If, however, the ink colour was red, the button to be pressed wats the button riagked to the written word, not the ink colour (e.g. if the word blue appears in red, the button brewas to be pressed). If the ink colour was blue, green or yellow, then the correct butfon pressed matched the ink colour. The word presented and its ink colour was randomly seteeted by the computer. Twenty practice attempts were allowed to ensure the participant fully understood the instructions. The Stroop task was also performed for 5 min during familiarization in visit 1. Participants were instructed to respond as quickly and accurately as possible. Visual feedback was given after each word in the form of correct or incorrect response, reaction time, and accuracy so far.

\section{Control}


The control condition consisted of watching a neutral documentary on cars and trains performed under the same conditions as the Stroop task. Participants were instructed to sit quietly in front of the computer screen for $60 \mathrm{~min}$.

\section{Familiarization Test and Time Trial}

During the first visit, participants underwent a familiarization exercise test. The exercise test was completed on a kayak ergometer (Speed Stroke Gym, Kayak-Pro, USA). Participants completed the time trial during each of the other 2 visits to the laboratory. A standardised warm-up was completed by all participants prior to each time trial using the same ergometer utitized for the familiarization visit. The time trial was then completed on the same ergometer. AtP the ergometers were fitted to replicate the same participants' positions. Participants were instructed to complete $2000 \mathrm{~m}$ as fast as possible. A fan was placed behind the timer at a standardized position and turned on at the end of the warm-up and was on during all time trial, and water wasprenided ad libitum. During visits 2 and 3, a researcher who was blind to the experimentatereatment received by the participants provided verbal encouragement throughout the test. Thise fesearcher was consistent within participants. Another researcher recorded power output, stroke rate and time at the end of 400,800, 1200,1600 and $2000 \mathrm{~m}$ of the time trial.

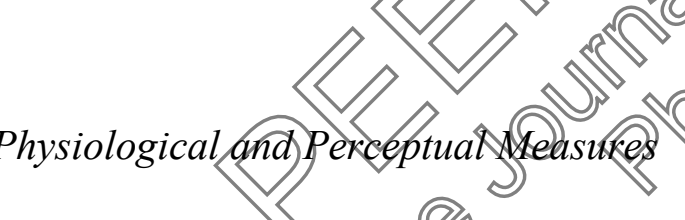

Capillary blood samplesave collected at rest, at completion of warm-up and time trial during visits 2 and 3. Samples were analysed immediately for blood lactate concentration using a portable blood lactate analyser (Lactate Pro, Arkray, Japan). During visits 2 and 3, heart rate was recorded at the end of the warm-up, and during the final $15 \mathrm{~s}$ of the 400, 800, 1200, 1600 and $2000 \mathrm{~m}$ of the time trial using a heart rate monitor fitted with a chest strap (Polar Team 2 and Polar T34 non-coded heart-rate transmitter, Polar, Finland). Rating of perceived exertion (RPE) was measured using the Borg 6-20 scale ${ }^{14}$. During visit 1, RPE was introduced to participants using standard procedures 
and anchoring ${ }^{14}$. During visits 2 and 3, RPE was measured at the end of the warm-up, and during the final $15 \mathrm{~s}$ of the $400,800,1200,1600$ and $2000 \mathrm{~m}$ of the time trial. At the appropriate time point, participants were asked to point on a large Borg 6-20 scale the number corresponding to their perception of effort defined as "the conscious sensation of how hard, heavy, and strenuous exercise is".

\section{Psychological Measures}

\section{Subjective workload}

The National Aeronautics and Space Administration Task Load Index (ASA-TLX) ${ }^{15}$ was used to assess subjective workload of the cognitive tasks. The NASA-T is composend six subscales: mental demand (How much mental and perceptual activity was required?) (Dhysical demand (How much physical activity was required?), temporal demand (How much time pressure did you feel due to the rate or pace at which the task occurred? performanca successful do you think you were in accomplishing the goals of the task set by the exgerimenter?), effort (How hard did you have to work to accomplish your levetof perfopmanc?) and(taystration (How irritating or annoying did you perceive the task?). Participants were asked to score each of the items on a scale divided into 20 equal intervals anchore by the bipolar desgetors high and low. This score was multiplied by 5 , resulting in a funal seore between 0 and 500 for each of the subscales. Regarding the subscale of success, the value on the sheet then inverted as mention in the instructions of the NASATLX [15].

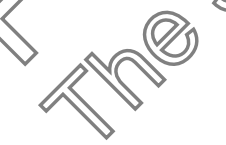

\section{Mood}

The Brunel Mood Scale (BRUMS) developed by Terry et al. ${ }^{16}$ was used to assess changes in mood from the beginning to the completion of the cognitive tasks of visits 2 and 3. This questionnaire, which is based on the Profile of Mood States, contains 24 items (e.g., angry, uncertain, miserable, tired, nervous, energetic) divided into six respective subscales: anger, confusion, depression, 
fatigue, tension, and vigor. The items are answered on a 5-point Likert scale $(0=$ not at all, $1=\mathrm{a}$ little, 2 = moderately, 3 = quite a bit, 4 = extremely), and each subscale, with four relevant items, can achieve a raw score in the range of 0 to 16.

\section{Motivation}

Motivation related to the time trial was measured using the success motivation and intrinsic motivation scales developed and validated by Matthews et al. ${ }^{17}$. Each scale consists of 7 items (e.g., "I want to succeed on the task" and "I am concerned about not doing as -well as can") scored on a 5 -point Likert scale $(0=$ not at all, $1=$ a little bit, $2=$ somemat, $3=$ very much, $4=$ extremely). Therefore, total scores for these motivation scales range between 0 an 8 .

\section{Statistical Analysis}

Data are presented as mean $\pm \mathrm{SD}$ unless otherwise stâted (Phe effects of mental fatigue on performance, physiological, biomechanical and perceptuaderesponses to exercise were tested using the magnitude-based inference (MB/)approael ${ }^{18}$ by statistic was the difference between the two comditions) (mental fatigue condition minus control condition). Chances that the true effect $(20 \%$ confidence limits) was harmful, trivial or beneficial were quantitatively/estimated and then d $s$ oussed using qualitative descriptors $(<1 \%$, almost certainly not; -59\%, hery unifiely; $325 \%$, unlikely; 25-75\%, possibly; 75-95\%, likely; 95-99\%, very likely; $>99 \%$, almos entainly). When an effect could be both positive and negative (that is with its confidence limits overlapping the thresholds for substantiveness more than $5 \%$ in both directions) was considered unclear. A smallest worthwhile (important) change for an outcome measure has to be defined a priori when the MBI approach is used; the smallest important change for time trial performance was set at $1 \%$ while a smallest standardized change was assumed to be $0.20^{20}$ for the other dependent variables (e.g. heart rate, RPE, etc.). The standardized difference in means (the mean difference divided by the between-subject standard deviation) was used as 
measure of effect size (ES) ${ }^{21}$. Threshold values of $0.20,0.60,1.20,2.0$ and 4.0 were considered as small, moderate, large, very large and extremely large respectively.

\section{Results}

\section{Performance and biomechanical measurements}

Kayaking performance (Fig 1) was almost certainly worsened after the mental fatigue task (552 \pm $30 \mathrm{~s})$ compared to control $(521 \pm 36 \mathrm{~s})$ with a mean increase in time to complete the $2000 \mathrm{~m}$ time trial by $5.7 \%(\mathrm{ES}=-1.03 \pm 0.25)$.

The effect of mental fatigue determined almost certainly lower power-qutputs at all the $400 \mathrm{~m}$ fractions (with moderate and large effect sizes) (Fig 6 a); only at the end of the $200 \mathrm{~m}$ time trial there was a very likely lower power output in the mental fatigue conditim( $(\mathrm{ES}=-0.53 \pm 0.24)$. Similarly stroke rate was very likely negatively affected during the 200 $\mathrm{m}$ time trial under mental fatigue (effect sizes from moderate to large) (Fig $6 \mathrm{~b})$.

\section{Psychological measurements}

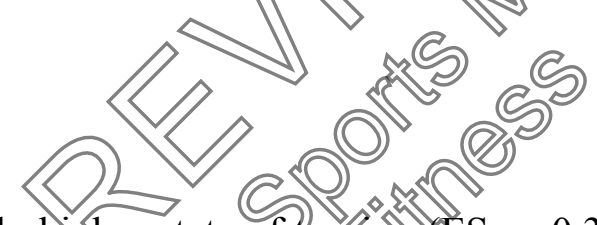

Apart from the possibly and likely higher state ofepsion (ES $=-0.30 \pm 0.42)$ and confusion (ES $=$ $0.44 \pm 0.48)$ respectively and the possibly loness state of anger $(\mathrm{ES}=0.39 \pm 0.47)$ prior to commencing the baseline strobp test during the experimental condition (Mental Fatigue), all the other items of mogd resulted Simbilaretween the two conditions (Fig 2 - Pre). On the contrary, almost certainly and yerele higher state of confusion $(\mathrm{ES}=0.97 \pm 0.44)$, anger $(\mathrm{ES}=0.86 \pm$ $0.55)$, depression $(\mathrm{ES}=0.85 \pm 0.63)$ and fatigue $(\mathrm{ES}=1.01 \pm 0.49)$, accompanied with a very likely lower state of vigor $(\mathrm{ES}=-0.89 \pm 0.65)$, were observed in the BRUMS completed after the mental fatiguing task (Fig 2 - Post). The experimental treatment affected the subjective workload (Fig 3) (effect statistics from possibly to almost certainly) resulting higher compared to the control condition with effect sizes for the single items ranging from moderate (physical demand, ES $=0.58$ $\pm 0.51)$ to very large (mental demand, $\mathrm{ES}=1.89 \pm 0.45$ ). A trivial effect statistic for intrinsic 
(experimental $21.9 \pm 2.5$ a.u, control $21.8 \pm 2.5$ a.u., $\mathrm{ES}=0.06 \pm 0.30$ ) and success (experimental $15.3 \pm 4.9$ a.u, control $15.5 \pm 4.5$ a.u., $\mathrm{ES}=-0.04 \pm 0.30)$ motivations was seen.

\section{Physiological and perceptual measurements}

Blood Lactate concentration (Fig 4) was possibly higher ( $\mathrm{ES}=0.15 \pm 0.20)$ at baseline during the experimental condition but resulted almost certainly lower $(\mathrm{ES}=-1.09 \pm 0.36)$ during the same condition at the end of the $2000 \mathrm{~m}$ time trial compared to control. A likely lower heart rate (Fig $5 \mathrm{a}$ ) with moderate effect sizes (ranging from $-0.28 \pm 0.25$ to $-0.44 \pm 0.30$ ) was recorded every $400 \mathrm{~m}$ during the experimental condition; heart rate at $2000 \mathrm{~m}$ was possibly lower with a moderate ES ($0.29 \pm 0.25$ ) during mental fatigue. On the contrary, RPE (Fig 5 b) was from possibly to almost certainly higher (with moderate effect sizes) every $400 \mathrm{~m}$ when the participants were under mental fatigue but at the end of the time trial where a possibly fower perception of effort was recorded (ES $=-0.33 \pm 0.44)$

\section{Discussion}

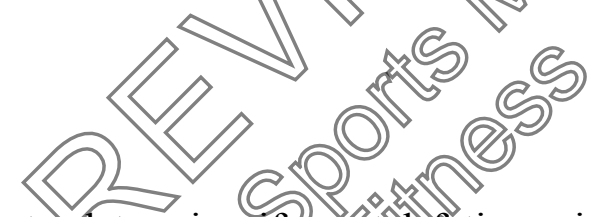

The main aim of this study was to determine ifment fatigue, induced by 60 min Stroop task would affect performance on $2000 \mathrm{~m}$ kayaking trime trial in young elite athletes. The outcome confirms our hypothesis that hintar(fatigug 9 poduced a detrimental effect on kayaking performance as we have observed infime, gower ouput and stroke rate during the time trial. As stated in a recent review and meta-analysisid a possible random error could explain the negative effects of cognitive tasks prior to physical performance in several studies published up to now. For this reason, our study, using a modern contemporary statistical approach ${ }^{18}$, showed a strong negative effect of mental fatigue on kayak endurance performance and contributes to the enrichment of the knowledge upon the relation between mental fatigue and physical performance. 
Participants showed the same level of motivation during the two conditions; this finding excludes any potential effect of this important component on performance, physiological and all the other psychological variables. Given the high level of athletes involved, we managed to keep similar high level of motivation through encouragement and in particular by underlining that performance would be compared with the ones from the others team mates ${ }^{23}$.

Mood assessed before the experimental and control treatment was nearly identical except for few items. In particular, tension and confusion seemed marginally higher the day of the experimental treatment compared to control while anger evidenced an opposite tendency on the same day. Although the chances for these differences were low (with small to moderate effect sizes), it can be hypothesized that they could be attributed to the uncertainty and concenn for the completion of the experimental task. Indeed, participants, due to the previous familiarization, were aware of the characteristics of the task and the relatively long time to spend for its cegomplishment. On the other way, results of the BRUMS after the experimental task wergente typical of a state of mental fatigue and consistent with previous literature ${ }^{4,5}$. Higherstates of confusion, tension, depression, fatigue and a lower vigor were evident signs of ment fatigue induced by the performance of a highly demanding cognitive task such as 60 minutes of the stroop color task. Furthermore, the NASA TLX questionnaire showee a nearly identical perceived physical demand on the two occasions but with/all the /other cognitive items higher during the experimental condition. Altogether, from psychological point of view, the experimental task effectively induced mental fatigue in the partieipaptis

Prior studies where the effect of mental fatigue on physical performance were investigated showed similar results to the present study for what concerns the psychological questionnaires ${ }^{4,5}$.

\section{Effect of mental fatigue on time trial performance and pacing}

As described above, mental fatigue was successfully induced in the participants the day of the experimental treatment and this led to a decrease in performance by almost $6 \%$. Previous research 
on the effect of mental fatigue on 3000 m running time trial ${ }^{24}$ showed a decrease in performance around $2 \%$. Similarly, other authors, showed that $5000 \mathrm{~m}$ running performance was decreased by about $5 \%$ after a response inhibition task ${ }^{5}$. In addition, a further study in which swimming was used as exercise modality reported the detrimental effect by $1.2 \%$ of mental fatigue on performance ${ }^{25}$. Although different kind of exercises (running and swimming versus kayaking), these seem to be the only studies where the effects of mental fatigue on whole body time trial performance were investigated.

It deserves to be highlighted that in the present study, compared to that of MacMahon et al. ${ }^{24}$ and Penna et al. ${ }^{25}$, there was a higher detrimental effect of mental fatigue despite a shorter duration of the mental fatigue task preceding the physical exercise $(60 \mathrm{~min}$ Stroop cotor task लersus 90 minutes AX CPT). There may be two reasons for this difference. Firstly, athough (tge Stroop task and the AX CPT task share similarities in terms of the cognitixy fundtions the target in the brain, they used different paradigms which suggest they may produce diffêren ffects in the brain ${ }^{26}$. So it can be speculated that 60 min of Stroop, well known for its streng inhibitory component ${ }^{26}$ may induce similar amount of mental fatigue as using $90 \% \mathrm{~min}$ (6) $\mathrm{AX}$ (20) Task. Furthermore, it cannot be excluded that 30 min of Stroop task prior toswammongerformance ${ }^{25}$ were not enough to cause high levels of mental fatigue.

However, another rêevani difference is the younger age of the participants of the present study (around 16 compared to 25 yeges old. This might suggest that young athletes are more mentally fatigable than adults ean be speculated that young individuals are less trained to tolerate cognitive tasks compared to adults; this is because adults received a larger bulk of cognitive stimuli during their lifespan ${ }^{27}$.

Pacing strategy was similar in the two conditions and was a parabolic-shaped pacing; this means that mental fatigue did not affect strategy used by athletes to pace themselves during the time trial. An additional reason why elite kayakers showed unaffected pacing strategy may be due to the fact that they are used to this type of exercise. 
Those results are in line with the one from MacMahon ${ }^{24}$ and Pageaux ${ }^{5}$. When the kayakers underwent the time trial in the mental fatigue condition were constantly exercising at a lower power output (Fig 6). Due to the characteristics of the kayaking and the ergometer used to simulate the $2000 \mathrm{~m}$ time trial, it was possible to detect that the lower power output entailed by the mental fatigue condition was caused by a lower stroke rate. It seems that mental fatigue influences negatively, at least in kayaking, the frequency of the actions. Those results are in line with a previous study ${ }^{28}$ showing that a combination of physical and cognitive fatigue affected cadence during a cycling time to exhaustion test.

Stroke rate is a paramount component of kayaking performance and if has to be kept as high as possible during the competition to keep a constant speed on the boat ${ }^{29}$. However higher stroke rate demands significantly higher power output from muscles ${ }^{29}$. Therefore if the stroke rate is impaired, subsequently also performance will be affected

As previous studies on mental fatigue and physical perforance have not measured pacing components such as stride and frequency in rumning or geäechanging and cadence in cycling, this is the first study, to the best of our knowledge, measing the effect of mental fatigue on pacing components during a time trial performance.

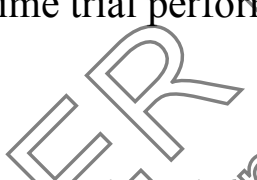

Effect of mental fatigue on physiologidal resporses to exercise and RPE

As expected heart rate and RRgencreas over time during the time trial and this is consistent with previous research $5,24,250$, differently to previous studies, in the present study a lower heart rate was encountered in the presence of mental fatigue. Considering the lower power output, it is evident that the physiological response was lower in the mental fatigue condition compared to the control. Moreover, blood lactate provided a similar trend across each visit and between the two groups in line with previous studies [3], showing that, at exhaustion, it was almost certainly higher in the control condition, most likely because of the higher power output produced. 
However this was not sufficient to reduce RPE: perception of effort is in large part dependent by the central motor command ${ }^{30}$ and therefore we should have expected a lower RPE but this was not the case. The main reason is that mental fatigue altered perception of effort as suggested in previous studies both during time trial and time to exhaustion ${ }^{3-5}$. The large effect of mental fatigue on perception of effort seen in the present study is suggested by the higher RPE in the mental fatigue condition.

The mechanism underpinning this finding has to be ascribed to the areas of the brain involved during the cognitive task (namely the Stroop color task performed prior to exercising) and the physical task (the 2000 m kayaking performance). As described in prexious works ${ }^{3-5,7}$, both types of tasks requires high level of effort and such effort (either physical oreonitires seems to share similar brain areas in terms of activation and neurotransmitters modulatign. Anterior cingulate cortex (ACC), motor cortex and supplementary motor area) (SMA) en to be heavily involved in generating perception of effort ${ }^{3}$. Thus, previgussy actiyation o⿺those areas using 60 minutes of cognitive task is likely to produce alterations in those areas, It is therefore evident that this sort of "deficiency" has an effect on the suecessive activation of thes area during the physical task. The result is a detrimental effect on the perception of effort (RE) during the physical task, that is a higher RPE for the same power output when timesto exhaustion is performed ${ }^{3}$ or a lower power output for the same RePE in timpe trians performance ${ }^{5}$.

In the presents sudy the huge gefect of mental fatigue caused by the cognitive task yielded an even worse and unexpected outeome: an even higher RPE with a lower power output during the experimental condition. In practical terms, as locomotor muscle fatigue "per se” decreases physical performance ${ }^{31}$, performing a cognitive task is a kind of fatiguing exercise for the brain that decreases physical performance. These two outcomes are both mediated by a higher perception of effort.

As suggested in previous study and confirmed by the results in the present study, RPE seems to ultimately mediate the outcome of performance. According to the psychobiological model 
proposed by Marcora and colleagues ${ }^{3,31-33}$ exercise performance in time trial tests is determined by five factors:

1. Potential motivation, defined as the maximum effort an individual would be willing to exert in a physical task ${ }^{34}$.

2. The Perception of Effort, defined as the conscious sensation of how hard, heavy, and strenuous exercise is ${ }^{32}$.

3. The subject's memory of the effort perceived during previous exercises of different intensities and durations.

4. The subject's knowledge of the total time trial time/distance cover, which refers to the awareness of the time, speed or power needed to complete the task and toplan the suitable strategy to complete the task in the shortest time.

5. The subject's awareness of the elapsed time/distance remaining, which refers to the feedback given on the time/distance remaining to complete the task.

If we consider that in the present study, motivation did wot change across the visits. Given that factors 3, 4 and 5 related to previous mowledge andexperience in the specific performance test did not impact performance through pacing as our elite young athletes have had multiple years of experience and practice in performing this specifielest. Perception of effort resulted to be the only parameter that ultinatey affected performance in the current study.

\section{Mental fatigue and erite athletes}

Results in the current study showed that mental fatigue affected time trial performance in elite young athletes. Previously, only one study has been published which compared effect of mental fatigue on both elite athletes vs amateur cyclists ${ }^{7}$. In that study mental fatigue induced by 30 min of Stroop task did not affect cycling performance in elite cyclists while, instead, impaired the performance in amateur ones. Our results are contradictory to those ones by Martin et al. ${ }^{7}$. A 
possible explanations could be that cyclists in the study by Martin and colleagues ${ }^{7}$ were exposed to only $30 \mathrm{~min}$ of Stroop test instead of $60 \mathrm{~min}$ as we did in our study. So it may be argued that 30 min of a cognitive demanding task may be enough to induce mental fatigue and produce a negative effect of performance in amateurs, while for elite athletes is probably not sufficient. It can be speculated that if cyclists in Martin`s study ${ }^{7}$ would be exposed to 60 min of Stroop task, that could have probably produced a detrimental effect in the time trial performance as we saw in our current study. In support of this theory, it is interesting to point out that in the study by Pageaux et al. ${ }^{5}$ the effect of 30 min of Stroop task entailed a decrease of 5\% in performance in amateur runners, while in the current present study elite athletes needed to undertake 60 min of Stroop task to produce a similar detrimental effect on the performance (about $6 \%$ ).

As speculated my Martin and colleagues ${ }^{7}$, elite athletes mayłbe more Pesiliegy to mental fatigue due to high intensity and volumes of training they are exposed oyer years or practice, due to a more selfregulated types of life (training routines, diet and \$o on and genetese factors.

\section{Conclusion}

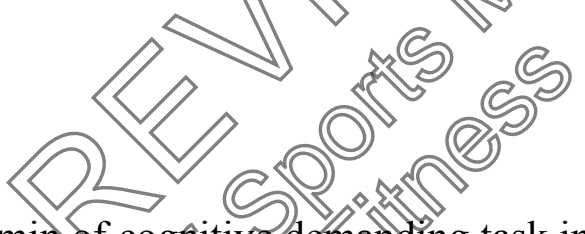

This investigation proved that 60 min of coghitive demanding task inducing mental fatigue affected kayaking performance in elite oung athletes. Thisestudy is the first of its kind to use kayaking as measure of sport performance and it may be beneficial for all scientists and coaches interested in the cognitive aspeet of kayak refated to performance. Moreover, the present study is the first to show that mental fatigte performance such as stroke rate as previously studies has never put attention on it. Lastly, this research is the first one using a young sample (under 16) of elite athletes to test the effect that mental fatigue has on their kayaking performance. 
Acknowledgements The authors would like to thank all the participants to the study for their valuable commitment especially during both the actual experimental treatment and all the effortful time trials.

Conflicts of interest The authors declare none conflicts of interest

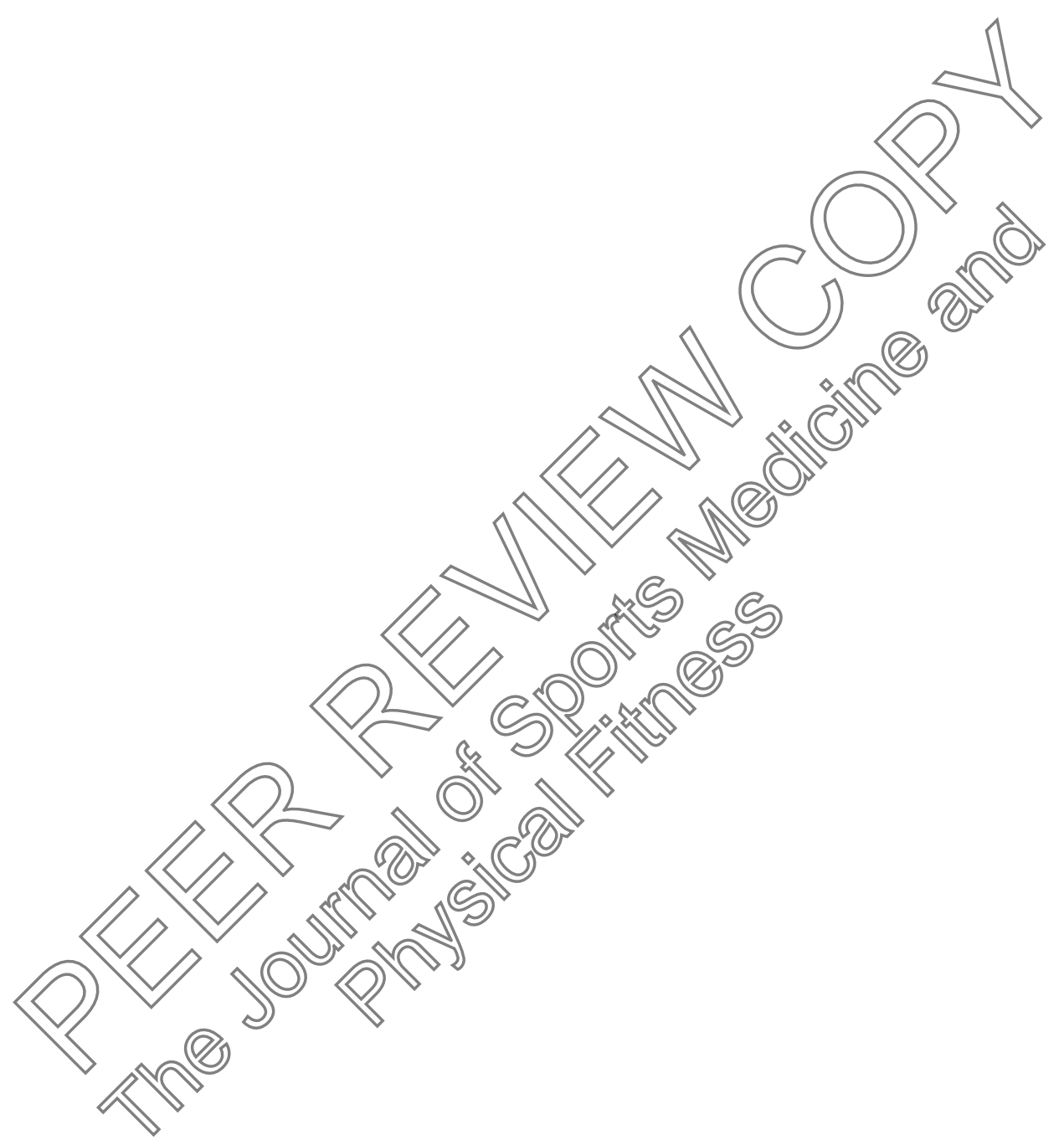




\section{References}

1. Walsh, V., Is sport the brain's biggest challenge? Curr Biol, 2014. 24(18): p. R859-R860.

2. Van Cutsem, J., et al., The Effects of Mental Fatigue on Physical Performance: A Systematic Review. Sports Med, 2017. 47(8): p. 1569-1588.

3. Marcora, S.M., W. Staiano, and V. Manning, Mental fatigue impairs physical performance in humans. J Appl Physiol (1985), 2009. 106(3): p. 857-64.

4. Pageaux, B. and R. Lepers, Fatigue Induced by Physical and Menta/Exertion Increases Perception of Effort and Impairs Subsequent Endurance Performance. Fyont Physiol, 2016. 7: p. 587.

5. Pageaux, B., et al., Response inhibition impairs subsequent self-pagelendurance performance. Eur J Appl Physiol, 2014. 114(5): p. 1095-1051

6. Smith, M.R., S.M. Marcora, and A.J. Coutts, Mental Fatgute Impairs Intermittent Running Performance. Med Sci Sports Exerc, 2015.47(8) 68290.

7. Martin, K., et al., Superior Inhibutory Controland Resistance to Mental Fatigue in Professional Road Cyetusts. PLoS One. 2016. 19(7): p. e0159907.

8. McMorris, T., et a1., Cognitive fatiguesffects on physical performance: A systematic review and meta-analysis. Physiol BBehav 26 18. 188: p. 103-107.

9. Michae, JS., K.B. Rooney, and R. Smith, The metabolic demands of kayaking: a review. J Sports Sci Med, 2008. 7(1): p. 1-7.

10. Shephard, R.J., Science and medicine of canoeing and kayaking. Sports Med, 1987. 4(1): p. 19-33.

11. Bishop, D., Physiological predictors of flat-water kayak performance in women. Eur J Appl Physiol, 2000. 82(1-2): p. 91-7. 
12. Gomes, B.B., et al., Gross efficiency and energy expenditure in kayak ergometer exercise. Int J Sports Med, 2012. 33(8): p. 654-60.

13. van Someren, K.A., G.R. Phillips, and G.S. Palmer, Comparison of physiological responses to open water kayaking and kayak ergometry. Int J Sports Med, 2000. 21(3): p. 200-4.

14. Borg, G., Borg's Perceived Exertion and Pain Scales. , ed. I.H.K. Champaign. 1998.

15. Hart, S.G.a.S., L.E., "Development of NASA-TLX (Task Load Index): results of empirical and theoretical research", in Human Mental Workload. 1988, P.A. Hancock|and N.

Meshkati: Amsterdam: North Holland. p. 139-183.

16. Terry, P.C., A.M. Lane, and G.J. Fogarty, Construct validity of the Profile of Mood States Adolescents for use with adults. Psychology of Sport and Exercise, 2003. f(2). p. 125-139.

17. Matthews, G., S.E. Campbell, and S. Falconer. Assessiment of motivotiggnal states in performance environments. in Proceedings of the Human Factors and Ergonomics Society Annual Meeting. 2001. Minneapolis Convention Cênter. $10.1177 / 154193120104501302$.

18. Batterham, A.M. and W.G. Hopkins, Mâking @iningfufinferences about magnitudes. Int J Sports Physiol Perform, 2006. 1(1): p $50-7$.

19. Hopkins, W.G., Aspreadsheet for analysigstraightforward controlled trials. . Sportscience, 2003.7 Available at: s.

20. Cohen, ..,Statistical Poger Analysis for the Behavioral Sciences (2nd Edition). 1988: Routledge.

21. Hopkins, W.G. A Scale of Magnitudes for Effect Statistics. 2002; Available at: http://www.sportsci.org/resource/stats/; Magnitudes].

22. McMorris, T., et al., Cognitive fatigue effects on physical performance: A systematic review and meta-analysis. Physiol Behav. 188: p. 103-107.

23. Boksem, M.A., T.F. Meijman, and M.M. Lorist, Mental fatigue, motivation and action monitoring. Biol Psychol, 2006. 72(2): p. 123-32. 
24. MacMahon, C., et al., Cognitive fatigue effects on physical performance during running. $\mathrm{J}$ Sport Exerc Psychol, 2014. 36(4): p. 375-81.

25. Penna, E.M., et al., Mental Fatigue Impairs Physical Performance in Young Swimmers. Pediatr Exerc Sci, 2018. 30(2): p. 208-215.

26. Stins, J.F., et al., Heritability of Stroop and flanker performance in 12-year old children. BMC Neurosci, 2004. 5: p. 49.

27. Glisky, E.L., Changes in Cognitive Function in Human Aging, in Brain Aging Models, Methods, and Mechanisms, D.R. Riddle, Editor. 2007, Taylor and Francis: Boca Raton (FL).

28. Keramidas, M.E., et al., Physiological and psychological determinants ofole-body endurance exercise following short-term sustained operations with outial sleep deprivation. Eur J Appl Physiol.

29. Baudouin, A. and D. Hawkins, A biomekhanicarevien factors affecting rowing performance. Br J Sports Med, 2002. 36(6): p. 306-402; disgussion 402.

30. Marcora, S., Perception of effort duking exercyse is independent of afferent feedback from skeletal muscles, heart,gnd lungs. J Appl Physjol (1985), 2009. 106(6): p. 2060-2.

31. Marcora, S.M., A. Bosio, and Harde Mroree, Locomotor muscle fatigue increases cardiorespikatoy responses and redinces performance during intense cycling exercise independently from metabolic Stress. Am J Physiol Regul Integr Comp Physiol, 2008. 294(3): p. R8t 4.83 .

32. Marcora, S.M. and W. Staiano, The limit to exercise tolerance in humans: mind over muscle? Eur J Appl Physiol, 2010. 109(4): p. 763-70.

33. Smirmaul, B.P.C., et al., The psychobiological model: a new explanation to intensity regulation and (in)tolerance in endurance exercise. Rev. bras. educ. fís. esporte, 2013. 27(2). 
34. Brehm, J.W. and E.A. Self, The intensity of motivation. Annu Rev Psychol, 1989. 40: p. 109-31.

\section{Figures captions}

Figure. 1 Effect of mental fatigue on 2000 m kayak performance. The black circles represent the mean values while the white circles the individual values. Data are presented as mean $\pm \mathrm{SD}$. The diamond (on the right hand side of the graph) represents the mean difference between the two conditions with the $90 \%$ confidence limits. \#\#\#\# almost certainly negative effect of mental fatigue on performance.

Figure. 2 The profile of mood state. The profile of phood state before (BRUMS Pre) and after (BRUMS Post) either the experimental intervention consisting 60 minutes of Stroop color task (Mental Fatigue) or the control condition consisting in watching 60 minutes of an emotionally neutral video (Control). Qualitative chanees thathe truevalue of the statistic is practically negative or positive: \# possibly, \#\# li ely \#\#\# ver fikely. When almost certainly. Whe chances are trivial or unclear no deseriptor is highlighted ôn the comparison present in the graph.

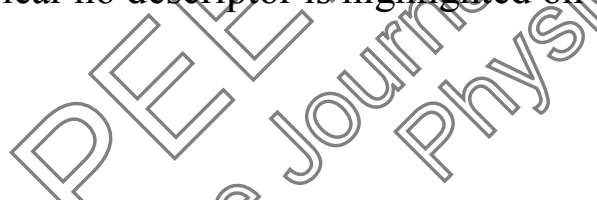

Figure. 3 The NASANA questionnaire. The effect of mental fatigue on the subjective workload assessed after the $2000 \mathrm{~m}$ kayak performance in both conditions (Mental Fatigue and Control). Qualitative chances that the true value of the statistic is practically negative or positive: \# possibly, \#\# likely, \#\#\# very likely, \#\#\#\# almost certainly. When the chances are trivial or unclear no descriptor is highlighted on the comparison present in the graph. 
Figure. 4 Blood Lactate concentration. Comparison of the blood lactate concentration between the experimental (Mental Fatigue) and control condition. Qualitative chances that the true value of the statistic is practically negative or positive: \# possibly, \#\# likely, \#\#\# very likely, \#\#\# almost certainly. When the chances are trivial or unclear no descriptor is highlighted on the comparison present in the graph.

Figure. 5 Heart rate and RPE during time trial. The effect of mental fatigue on heart rate (panel a) and perception of effort (panel b) assessed every 400m during the $2000 \mathrm{~m}$ kayak performance. Qualitative chances that the true value of the statistic is practically negative or posituke: \# possibly, \#\# likely, \#\#\# very likely, \#\#\# almost certainly. When the chances are frevial or unclear no descriptor is highlighted on the comparison present in the graph.
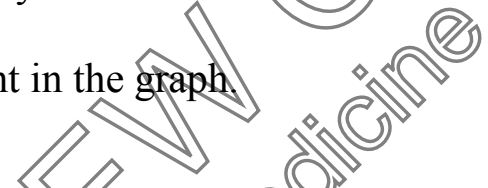

Figure. 6 Biomechanical parameters during time trial. The effectef mental fatigue on power output (panel a) and stroke rate (panel b) assessed ever $400 \mathrm{~m}$ daiting the $2000 \mathrm{~m}$ kayak performance. Qualitative chances that the true value of the stafistic is practically negative or positive: \# possibly, \#\# likely, \#\#\# very likely, \#\# almgstcertainger When the chances are trivial or unclear no descriptor is highlighted on the confarison present in the graph.

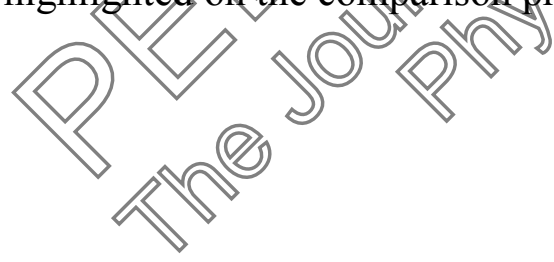




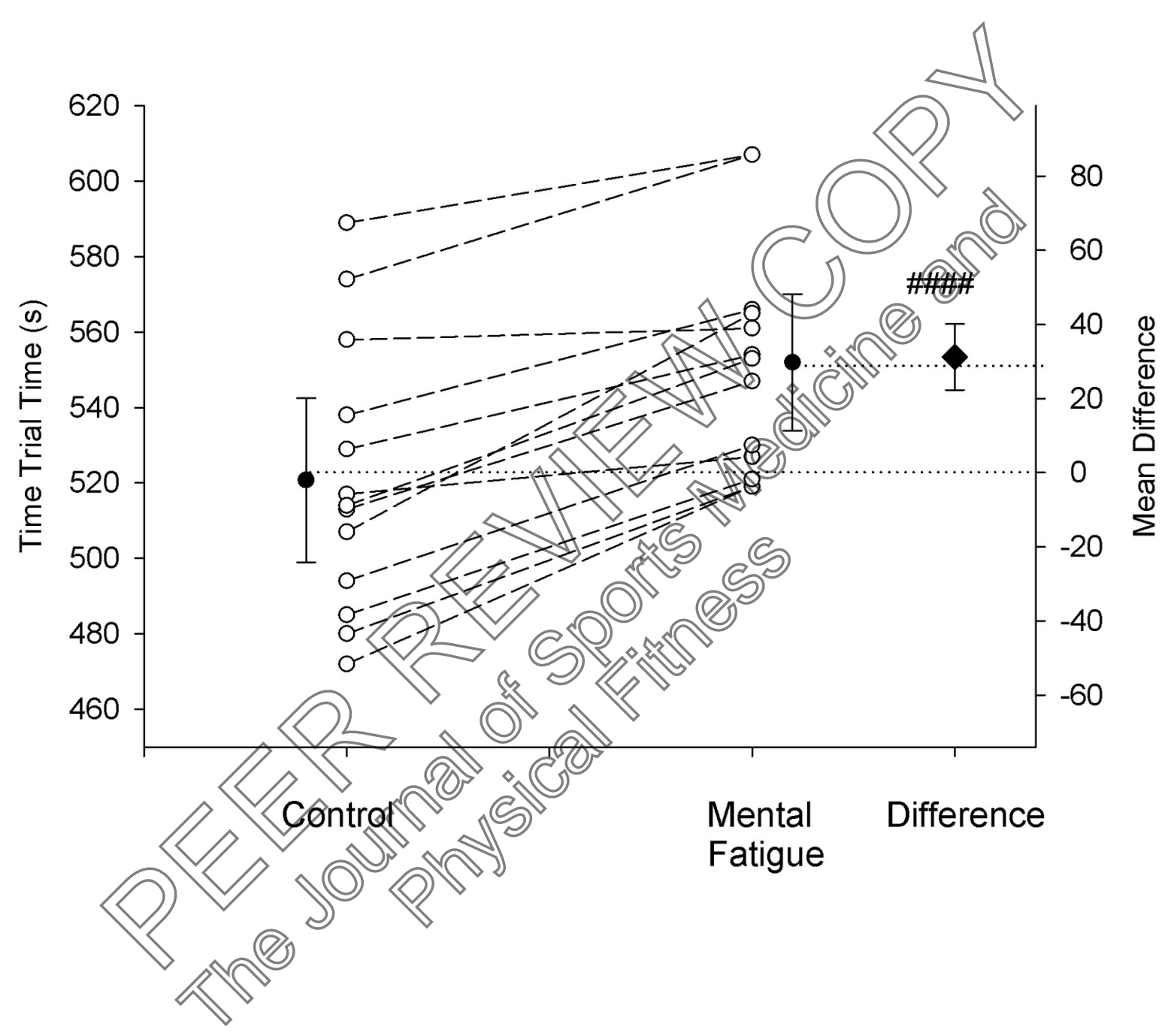

Page 25 of 30 


\section{(BRUMS Pre)}
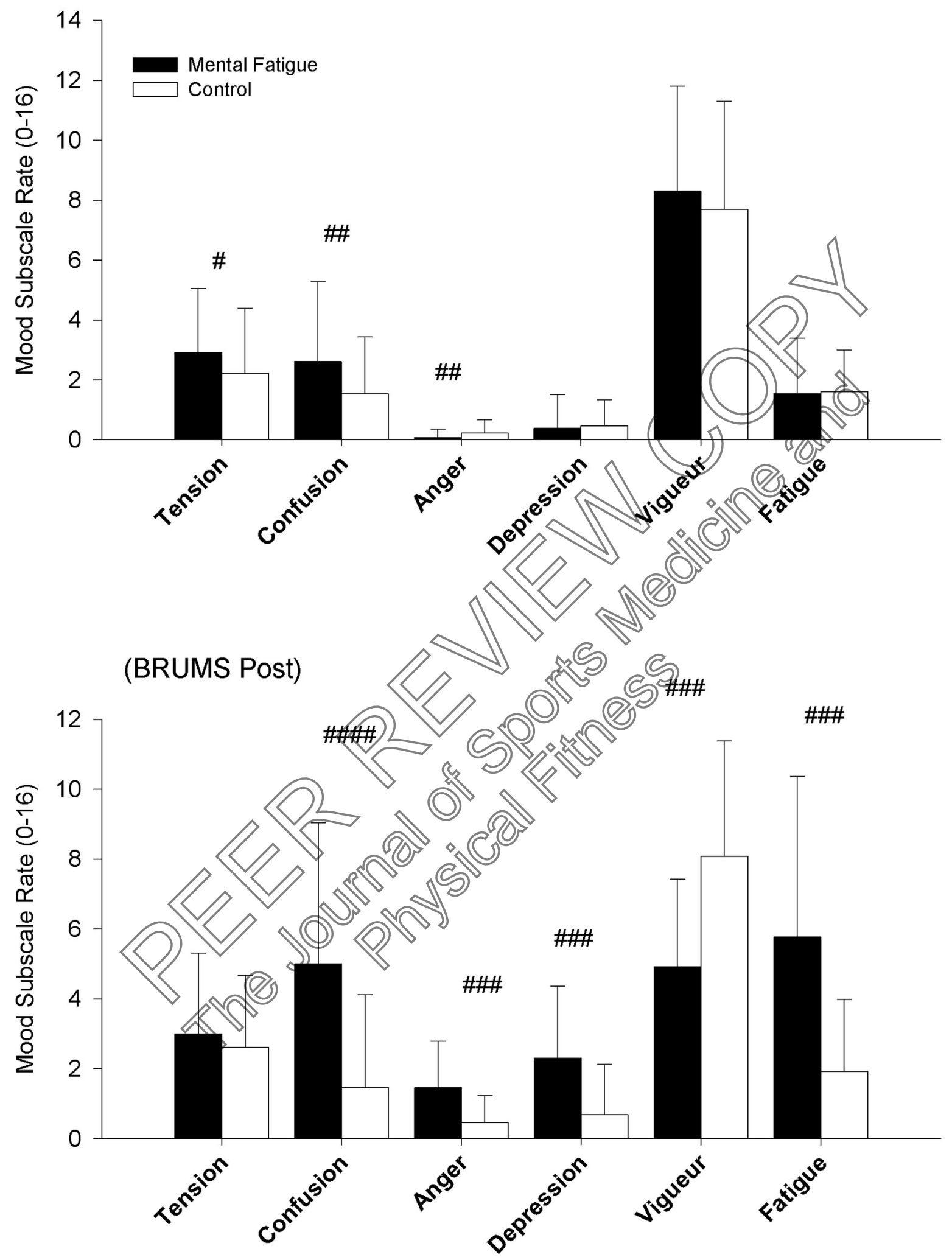

Page 26 of 30 


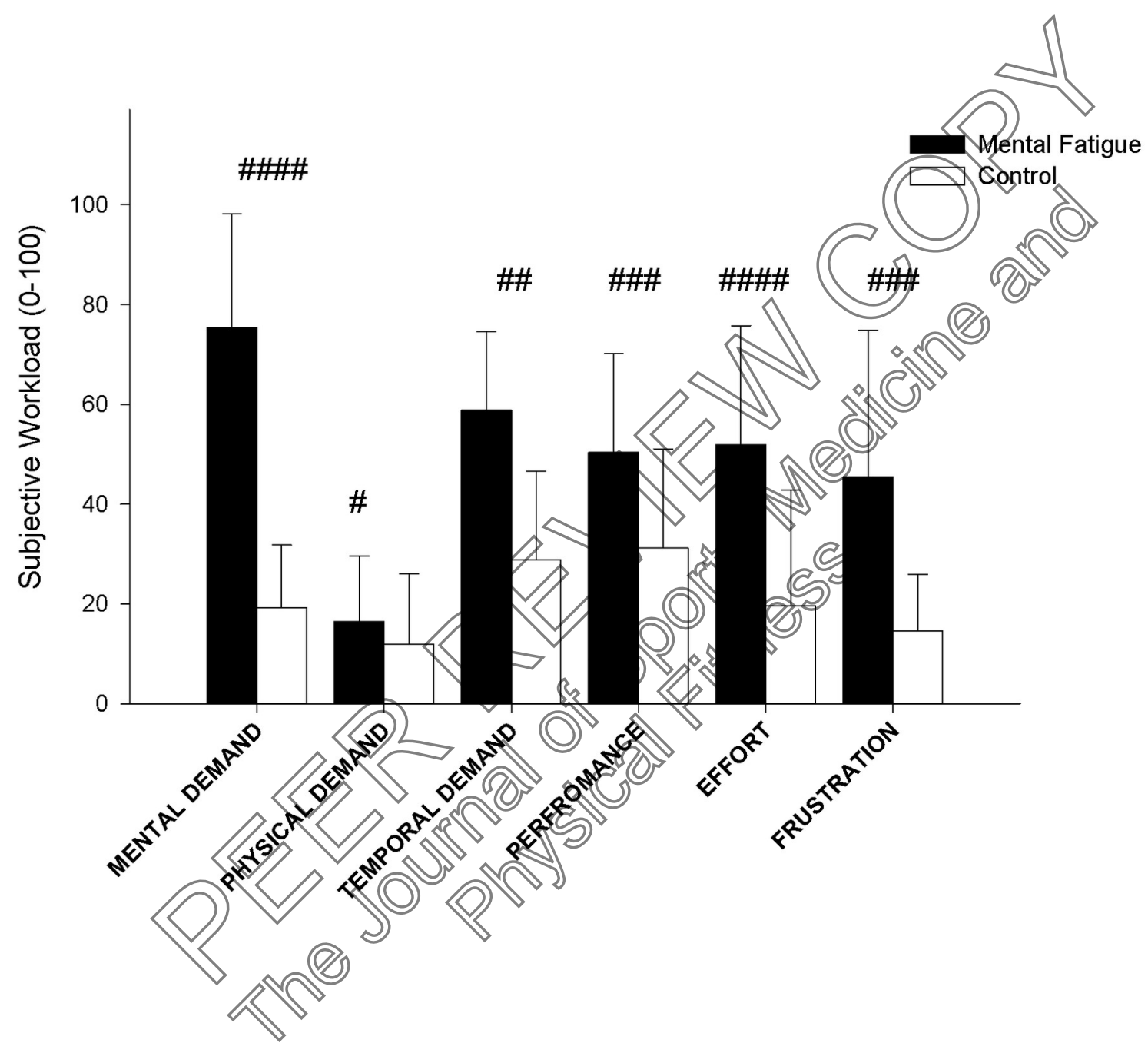




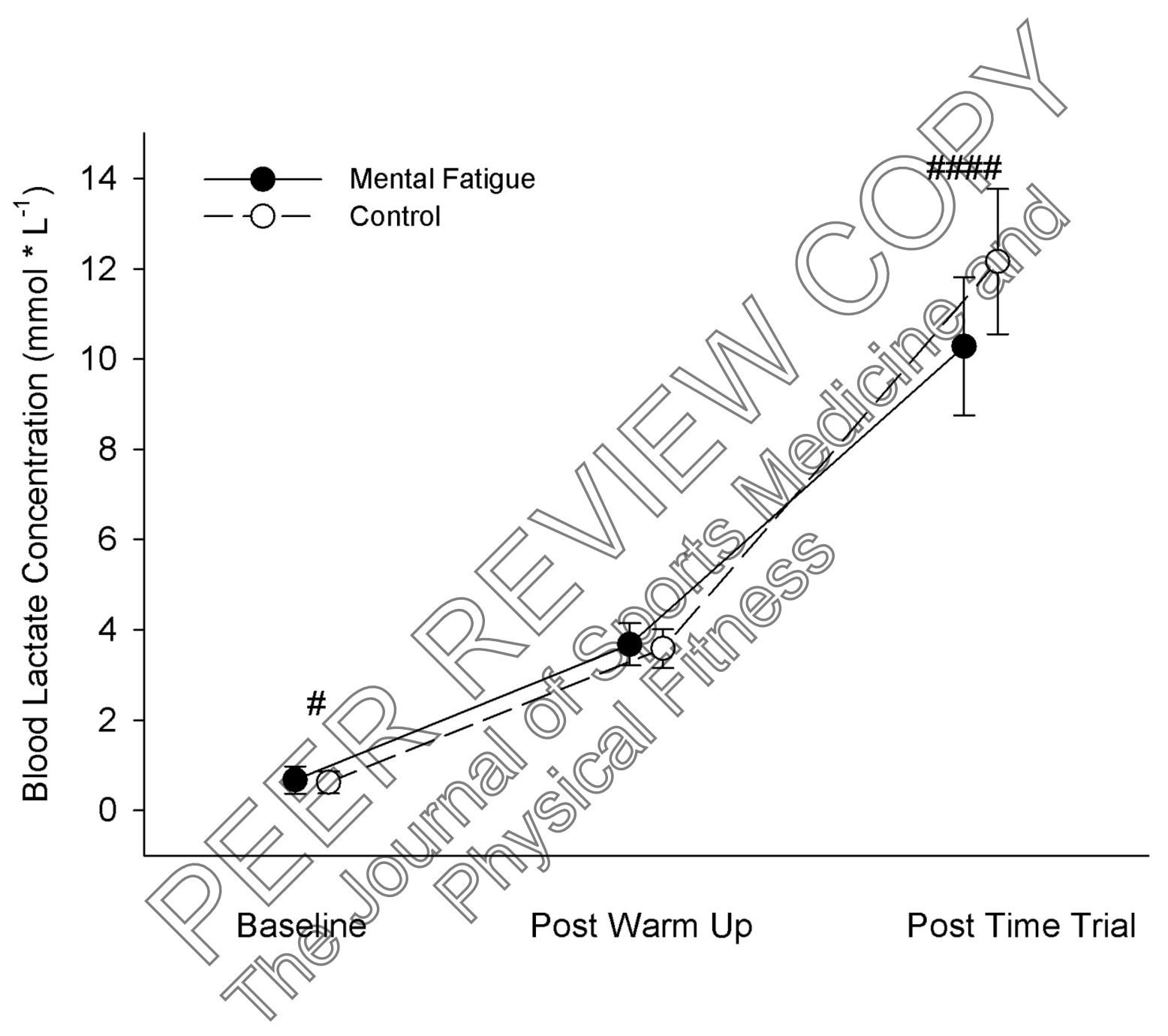

Page 28 of 30 
(a)

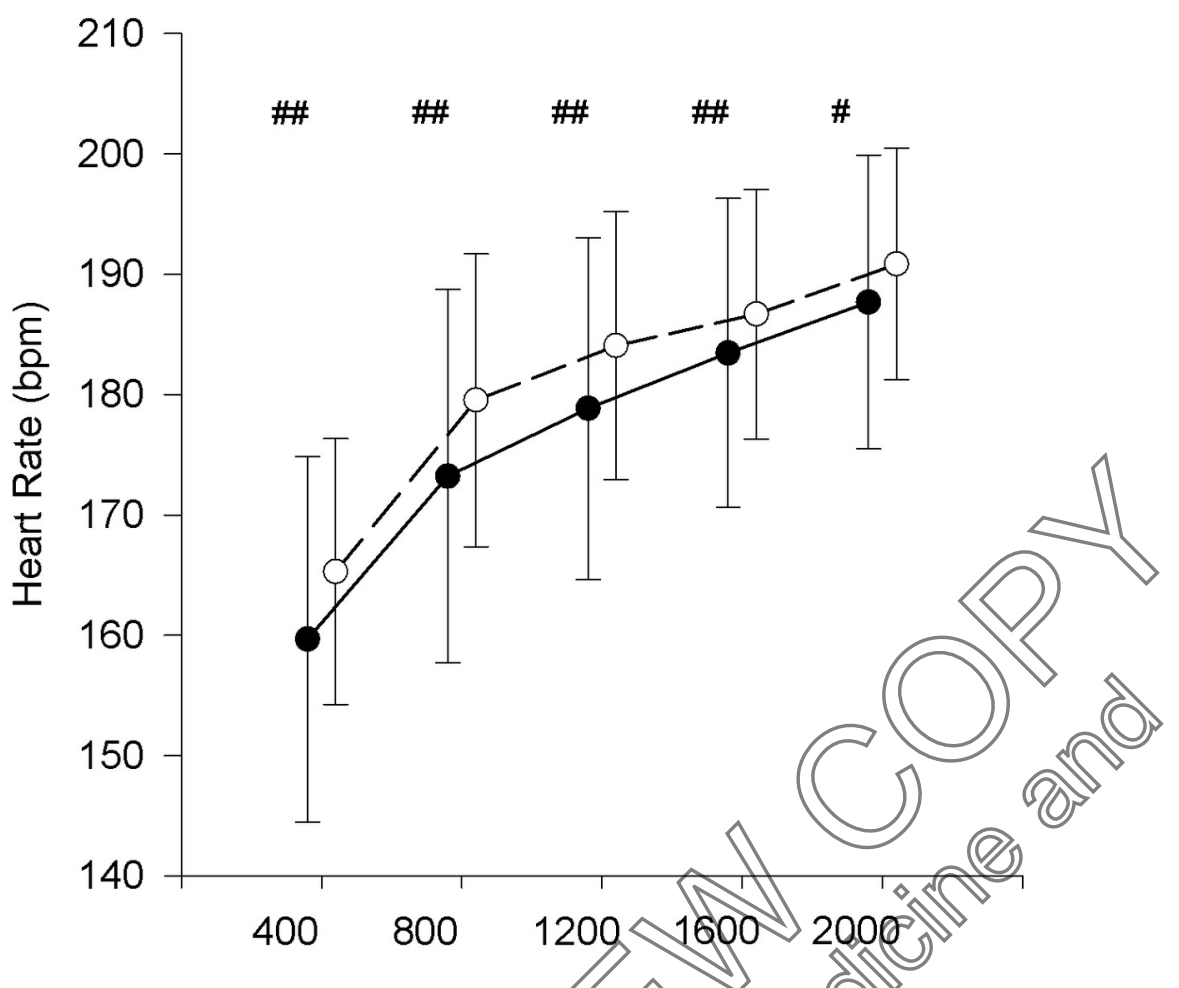

(b)
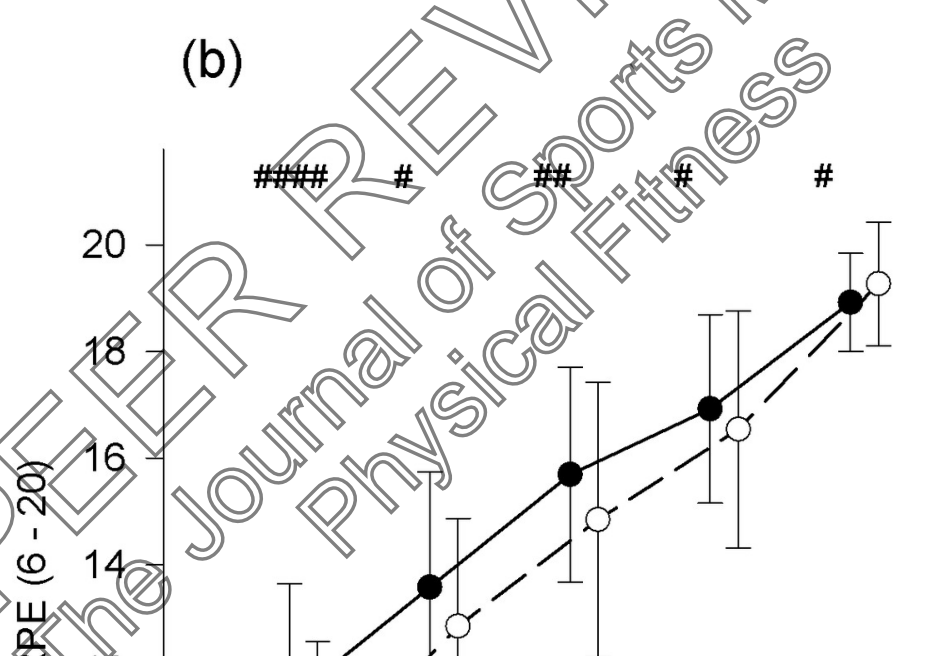

怔

近 
(a)

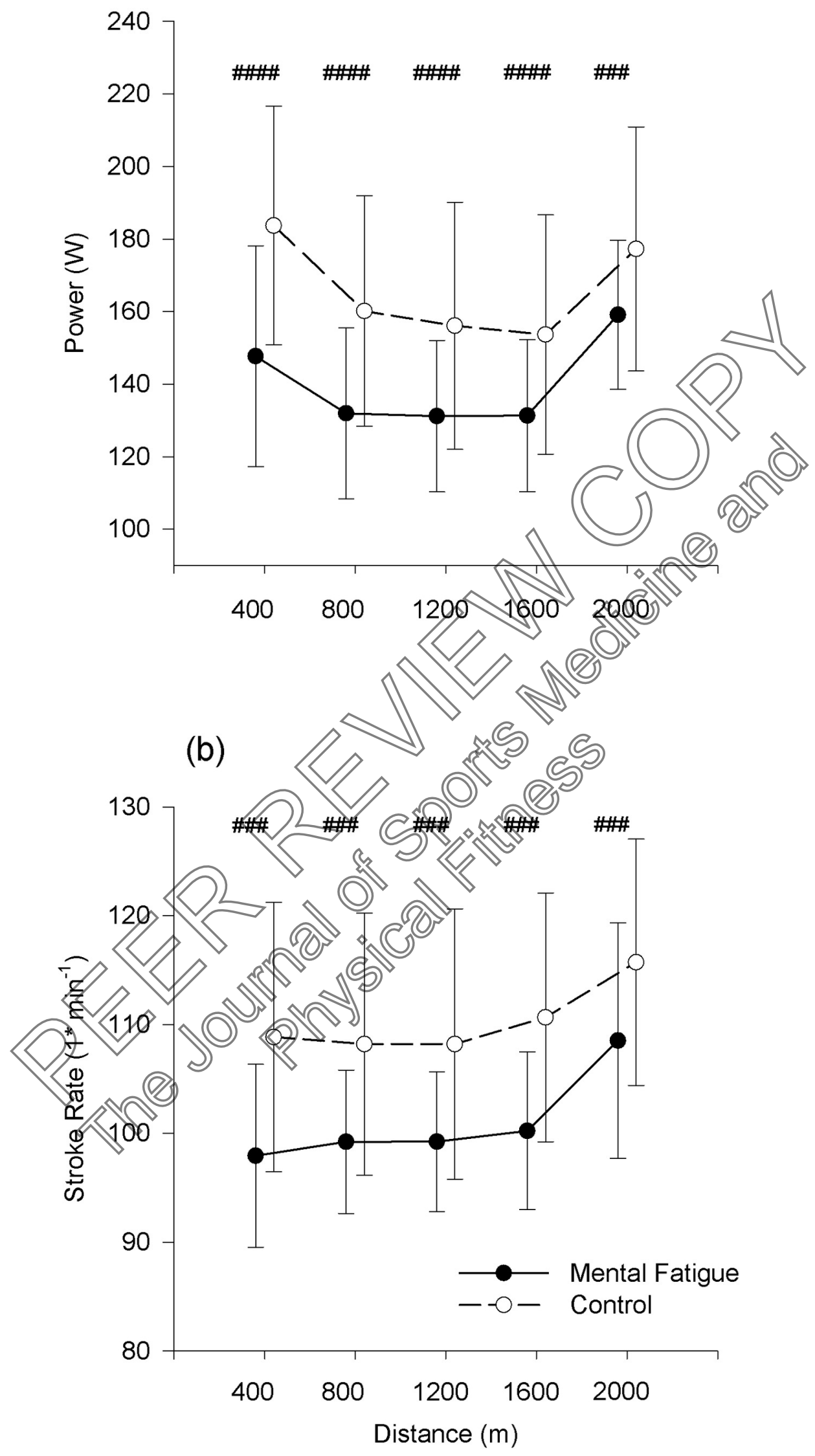

